\title{
Identification of a diffuse form of hyperinsulinemic hypoglycemia by 18 -fluoro-L-3,4 dihydroxyphenylalanine positron emission tomography/CT in a patient carrying a novel mutation of the HADH gene
}

\author{
Stefania Di Candia*, Alessandra Gessi ${ }^{1, *}$, Gino Pepe $^{2}$, Paola Sogno Valin, Eleonora Mangano ${ }^{1}$, Giuseppe Chiumello, \\ Luigi Gianolli $^{2}$, Maria Carla Proverbio ${ }^{1}$ and Stefano Mora ${ }^{3}$ \\ Department of Pediatrics, San Raffaele Scientific Institute, Vita-Salute San Raffaele University, Via Olgettina 60, 20132 Milan, Italy, ${ }^{1}$ Department of \\ Science and Biomedical Technologies (DiSTeB), University of Milan, Via Fratelli Cervi 93, 20090 Segrate, Milan, Italy, ${ }^{2}$ IBFM-CNR, Department of \\ Nuclear Medicine, San Raffaele Scientific Institute, University of Milan Bicocca, Via Olgettina 60, 20132 Milan, Italy and ${ }^{3}$ Laboratory of Pediatric \\ Endocrinology, San Raffaele Scientific Institute, Via Olgettina 60, 20132 Milano, Italy
}

(Correspondence should be addressed to S Mora; Email: mora.stefano@hsr.it)

*(S Di Candia and A Gessi contributed equally to this work)

\begin{abstract}
Objective: Congenital hyperinsulinism is the most common cause of persistent hypoglycemia in infancy (HI), leading to severe neurologic disabilities if not promptly treated. The recent application of positron emission tomography (PET)/computed tomography (CT) scanning with 18-fluoro-L-3,4 dihydroxyphenylalanine improved the ability to distinguish the two histopathologic forms of HI (focal and diffuse), whose differentiation heavily influences the therapeutic management of the patient.

Case report: We describe the case of a patient presenting with severe hypoglycemia from infancy. High concentration of insulin suggested the diagnosis of congenital hyperinsulinism. No metabolic disorders related to amino acid, organic acids or fatty acid oxidation were detected. Medical treatment was able to obtain a satisfactory metabolic response.

Results: The patient underwent PET/CT scanning, revealing a diffuse form of the disease. The absence of mutations in KCNJ11 and ABCC 8 genes (responsible for $50 \%$ of $\mathrm{HI}$ cases), and whole genome single nucleotide polymorphisms analysis by microarray suggested the HADH gene as a likely candidate. Sequence analysis revealed a novel homozygous nonsense mutation (R236X) in HADH gene.

Conclusions: This case indicates that mutations of the HADH gene should be sought in hyperinsulinemic patients in whom diffuse form of HI and autosomal recessive inheritance can be presumed when KCNJ11 and $A B C C 8$ genes mutational screening is negative, even in the absence of altered organic acids and acylcarnitines concentration.
\end{abstract}

European Journal of Endocrinology 160 1019-1023

\section{Introduction}

Congenital hyperinsulinism is the most common cause of persistent hyperinsulinemic hypoglycemia in infancy (HI). It occurs as a consequence of an unregulated hypersecretion of insulin by pancreatic $\beta$-cells, leading to the uptake of glucose by peripheral tissues, and to an inhibition of glycogenolysis, gluconeogenesis, lipolysis, and ketogenesis (1). An early and aggressive management of patients with congenital hyperinsulinism is mandatory to avoid brain damage and subsequent neurologic disabilities.

Two histopathologic lesions, with different molecular bases, characterize the focal and diffuse forms of the disease. The focal form is sporadic and due to the coexistence of a paternally inherited mutation in one of the genes encoding the subunits of the $\beta$-cell ATP-sensitive potassium channel (ABCC 8 and KCNJ11) and somatic loss of maternal $11 \mathrm{p} 15$ alleles within a limited region of the pancreas. The diffuse form is most frequently inherited as an autosomal recessive trait (1). Most cases are due to mutations in the $A B C C 8 / K C N J 11$ genes, but recently a rarer form of autosomal recessive HI (OMIM \# 609975), caused by mutations in short-chain 3 hydroxyacyl coenzyme A dehydrogenase gene (HADH entrez gene ID 3033), has been described (2-4). The HADH enzyme catalyzes the penultimate reaction in the fatty acid $\beta$-oxidation and its mutations result in a form of $\mathrm{HI}$ characterized by the presence of 3-hydroxyglutaric acid in urine and raised plasma levels of 3-hydroxybutyryl-carnitine (2). Children with this type of hyperinsulinism have no signs 
of hepatic dysfunction, cardiomyopathy or effects on skeletal muscle (3) that are typically present in patients with all other defects in fatty acid oxidation. The clinical presentation is heterogeneous, with either mild late onset hypoglycemia or severe neonatal hypoglycemia that is responsive to medical therapy with diazoxide. The mechanism leading to altered insulin secretion due to $H A D H$ gene mutations is still unclear.

We describe a patient carrying a novel mutation of the $H A D H$ gene, who underwent positron emission tomography (PET)/CT scanning and genome-wide single nucleotide polymorphism (SNP) analysis by microarray.

\section{Case report}

The propositus is an Italian boy born from consanguineous parents (Fig. 1). He was born at 40 weeks of gestation, and his birth weight was $3900 \mathrm{~g}$. He fed poorly from birth and he was described as lethargic. At the age of 2 months, he showed a fixed stare for a few minutes, right rotation of the head, and clonic movements of upper limbs. A neurologic disease was excluded. A blood glucose concentration of $0.94 \mathrm{mmol} / \mathrm{l}$ was measured. During a subsequent palpebral clonic episode that lasted about $30 \mathrm{~s}$, the patient's blood glucose concentration was $1.22 \mathrm{mmol} / \mathrm{l}$. He received a bolus of $20 \%$ glucose $(2 \mathrm{ml} / \mathrm{kg})$ and his glycemia rose to $7.16 \mathrm{mmol} / \mathrm{l}$. Further investigations performed during an episode of hypoketotic hypoglycemia (glycemia $2.38 \mathrm{mmol} / \mathrm{l}$ ) showed inappropriately elevated serum insulin concentration $(8.43 \mathrm{mUI} / \mathrm{ml})$. A glucose infusion rate of $8 \mathrm{mg} / \mathrm{kg}$ per $\mathrm{min}(0.43 \mathrm{mmol} / \mathrm{kg}$ per $\mathrm{min})$ was initially necessary, and after $24 \mathrm{~h}$ it was decreased due to the good response. A $5 \mathrm{mg} / \mathrm{kg}$ per min $(0.27 \mathrm{mmol} / \mathrm{kg}$ per $\mathrm{min})$ glucose infusion rate was sufficient to maintain blood glucose between 3.33 and $5.3 \mathrm{mmol} / \mathrm{l}$. He was evaluated for inborn errors of metabolism, and no metabolic disorders related to amino acid, organic acids or fatty acid oxidation was detected.

The clinical characteristics suggested the diagnosis of hyperinsulinemic hypoglycemia, and therefore the proband was referred to our centre for appropriate treatment. The patient was initially treated with
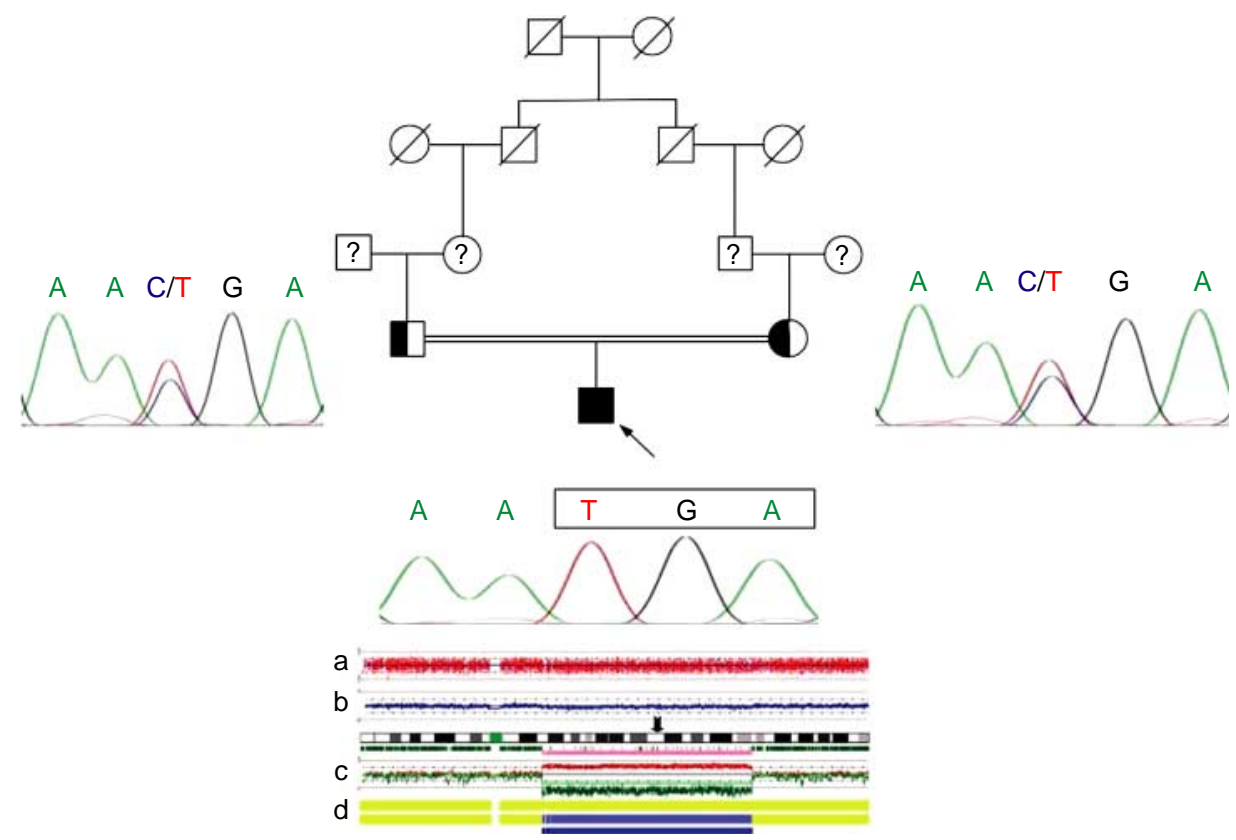

Figure 1 Pedigree and identification of $H A D H$ gene mutation in the $\mathrm{HI}$ family. Automated sequence analysis of PCR amplified genomic DNA from the affected subject shows a homozygous $C$ to T transition changing Arg to STOP codon at position 236 of the synthesized protein (R236X). Unaffected parents are heterozygous for the same mutation. Below, map of DNA analysis of patient's chromosome 4 is shown. DNA was analyzed on Affymetrix GeneChip Human Mapping 250K Nsp Arrays. Whole-genome DNA profile was assembled using Copy Number Analyzer for Affymetrix GeneChip software (CNAG, v3) and each sample was compared with a pool of normal controls (48 HapMap samples available on Affymetrix website). Genotyping quality control and pedigree check in the family showed call rates $>99 \%$ and Mendelian errors $<0.1 \%$. Chromosome 4 map is shown from $p$ to $q$ end (from left to right). (a) Red dots represent single SNP copy number signals on log scale; (b) total copy number values averaged on adjacent 10 SNP; (c) copy number values for each allele; bars in the middle represent heterozygous genotype calls and homozygous calls between the sample and normal controls and (d) the two bars at the bottom represent the color-coded visualization of total copy number status and LOH. Copy Number Neutral LOH stretch from $4 q 13.2$ to 4 q31.22 (from 68252878 to 147336231 bp according to UCSC Genome Browser, Human Assembly May 2004) observed in $\mathrm{CHI}$ affected patient and containing at $4 \mathrm{q} 25$ the already known $\mathrm{HI}$ causative gene HADH (Entrez gene ID 3033) is shown (arrow). Full colour version of this figure available via http://dx.doi.org/10.1530/EJE-08-0945. 
diazoxide (17 mg/kg per day) leading to a satisfying clinical response. Diazoxide requirement to maintain normal blood glucose and insulin concentrations was progressively reduced during follow-up, and it stabilized to a daily dose of $5.6 \mathrm{mg} / \mathrm{kg}$. Two attempts to interrupt treatment were made, but asymptomatic hypoglycemia (glucose concentration: $1.16 \mathrm{mmol} / \mathrm{l}$; insulin concentration: $10 \mathrm{mU} / \mathrm{ml}$ ) occurred 3-4 days after suspension.

Mutation scanning of KCNJ11 and ABCC 8 genes was performed, as previously described (5), but no mutations were found in the coding region of the two genes.

The absence of mutations in KCNJ11 and ABCC8 genes prompted the search for new susceptibility loci using genome-wide 250K SNP mapping array technology (Affymetrix, Santa Clara, CA, USA). The family profiles did not show any chromosomal aberrations, but we found a long contiguous trait of homozygosity of $79 \mathrm{Mb}$ on the patient's chromosome 4 (4q13.2-4q31.22, Fig. 1). Proband's parents were heterozygous in the same chromosomal segment indicating homozygous by descent trait nature. Additionally, transmission analysis of parents' haplotypes in the homozygous stretch excluded the presence of uniparental disomy (autozygosity). The autozygous segment contains 312 known genes including the $H A D H$ gene located at $4 \mathrm{q} 25$. Consequently, we sequenced the complete coding region of the $H A D H$ gene, including intron-exon boundaries. A novel homozygous mutation was identified at codon 236 $(706 \mathrm{C}>\mathrm{T})$ in exon 6 of the patient's HADH gene, resulting in the novel nonsense mutation R236X, while the parents were found heterozygous for this mutation (Fig. 1). To verify the effect of the nonsense mutation on $H A D H$ transcription, a PCR product covering exons 3-8 was amplified from reverse-transcribed peripheral lymphocytes mRNA using appropriate primer pairs. Agarose gel electrophoresis analysis of amplified product yielded an amplicon of $622 \mathrm{bp}$ which was directly sequenced, showing the skipping of the mutated exon 6 (data not shown).

At the age of 7 years, the patient was readmitted to our center for a diagnostic re-evaluation and he underwent PET/CT scan analysis with 18-fluoro-L-3,4 dihydroxyphenylalanine ( $\left[{ }^{18} \mathrm{~F}\right]$-DOPA) PET/CT to differentiate the HI form. Therapy with diazoxide was suspended 3 days before PET/CT scan. The imaging investigation included an abdominal ultrasound, abdominal CT, and wholebody $\left[{ }^{18} \mathrm{~F}\right]$-DOPA PET/CT. There were no intrapancreatic lesions identified in the ultrasound or CT examinations. PET/CT demonstrated intense and homogenous uptake of $\left[{ }^{18} \mathrm{~F}\right]$-DOPA (Fig. 2), thus suggesting a diffuse form of congenital hyperinsulinemia. This finding indicates increased metabolic activity in the islets, similar to that observed in patients with $\mathrm{HI}$ due to mutations in the

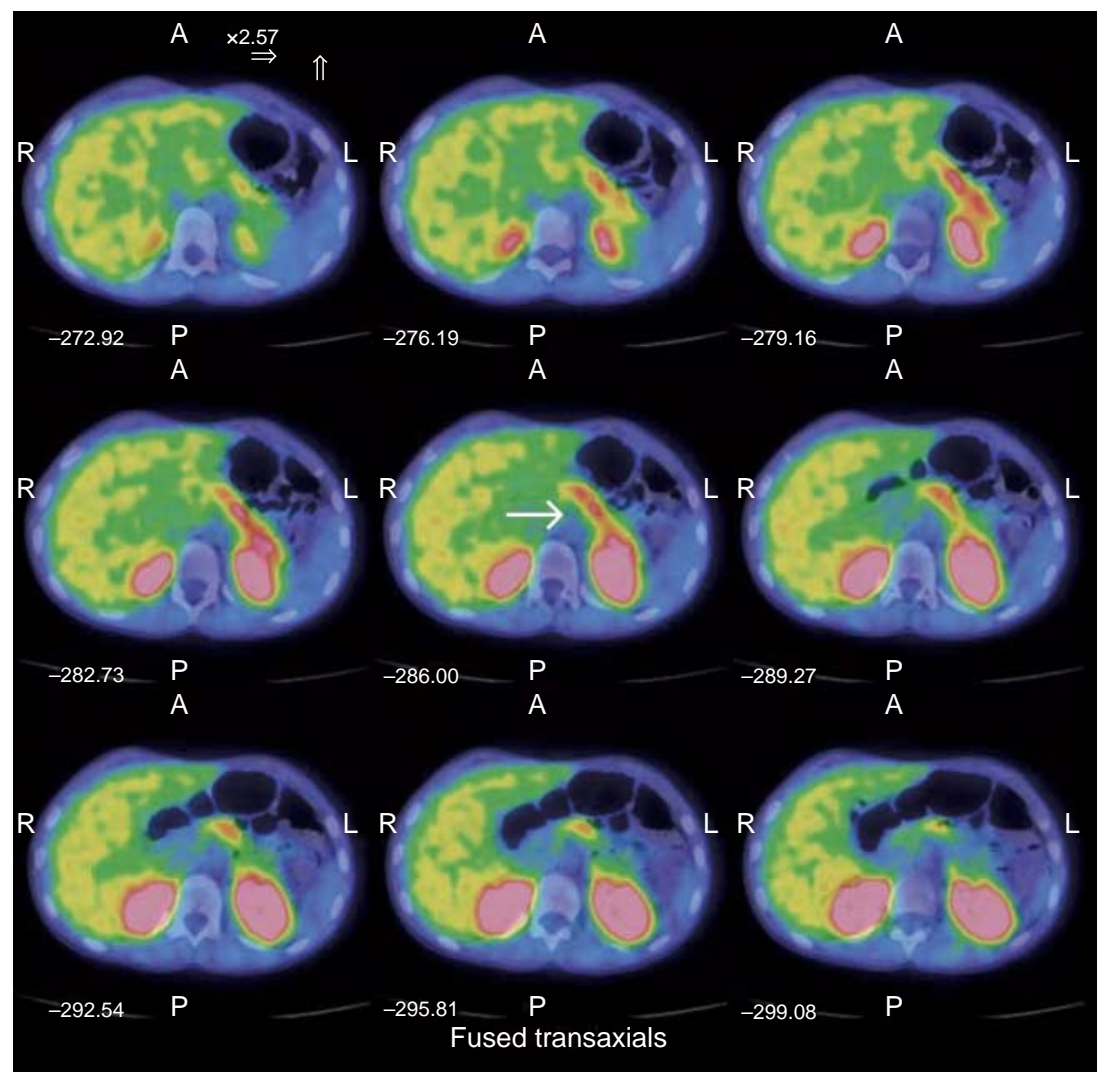

Figure 2 Transaxial PET/CT slices of upper abdomen, showing diffuse $\left[{ }^{18} \mathrm{~F}\right]-\mathrm{DOPA}$ uptake in the pancreatic gland (arrow). Intensity of uptake of $\left[{ }^{18} \mathrm{~F}\right]$-DOPA by the islets was equivalent to that observed in patients with defects in pancreatic ATPsensitive $\mathrm{K}^{+}$channels (6). Full colour version of this figure available via http://dx.doi.org/10.1530/EJE-08-0945. 
ABCC8 and KCNJ11 genes (6). After the examination, the patient restarted the therapy with diazoxide because of asymptomatic hypoglycemia.

Urine metabolites were re-evaluated showing an elevated excretion of 3-hydroxybutyric acid, a slight rise of dicarboxylic aciduria, while the level of acylcarnitine in blood was normal.

\section{Discussion}

We report the first case of a hyperinsulinemic patient bearing a nonsense mutation (R236X) of HADH gene. Mutations of the HADH gene causing $\mathrm{HI}$ have been described in three other families of different ethnic backgrounds (2-4): a missense mutation P258L, a six base pair deletion at the start of HADH exon 5 and a splice site mutation IVS6-2.

Age at onset of the reported cases varied from a few days to 4 months (2-4). Hypoglycemia led to seizures in two cases $(3,4)$, and to hypotonia and cyanosis in one (2). Blood glucose concentration at diagnosis ranged from 0.8 to $1.4 \mathrm{mmol} / \mathrm{l}(2-4)$. Our patient presented with a fixed stare and clonic movements at the age of 2 months, with a blood glucose concentration of $0.94 \mathrm{mmol} / \mathrm{l}$, indicating a highly variable clinical presentation of the disease. A common finding in all cases, including the present one, was the good response to medical treatment.

Unlike all other reported cases (2-4), neither specific increases of hydroxybutyryl-carnitine in blood nor of urinary 3-hydroxyglutarate were observed in our patient. It is difficult to interpret these findings. However, in vitro studies that silenced $H A D H$ gene expression in $\beta$-cells showed increased insulin release, which was not caused by increased rate of glucose metabolism or inhibition of fatty acid oxidation (7). Moreover, a similar case has been recently communicated (8). Elevated levels of urinary 3-hydroxybutyric acid, and slightly increased dicarboxylic aciduria were evident at his diagnostic re-evaluation. The HADH protein is known to act in a common branch of the cyclically organized $\beta$-oxidation pathway, converting L-3-hydroxyacyl-CoA of variable chain length to their corresponding 3-ketoacyl-CoA; the mitochondrial $\beta$-oxidation role in the regulation of glucose-induced insulin release has been examined in a recent work (7), suggesting that this enzyme could play a regulatory role between glucose metabolism and acyl-coA-derived signals. This view is supported by a moderate increase in glucose-induced $\mathrm{NADH}$ in severely $\mathrm{HADH}$ suppressed cells, a glucose-induced accumulation of L-3-hydroxybutyryl-CoA, the unbalance between HADH expression and L-3-hydroxyacyl profiles, and finally the complex influence of HADH expression on $\beta$-oxidation (7). The urinary metabolic profile of our patient is consistent with a slow down of $\beta$-oxidation at level of $\mathrm{HADH}$, leading to increased accumulation of medium and long L-3-hydroxyacyl-CoA species, and upstream long-chain acyl-CoAs, which stimulate insulin release (9). Long chain-acyl-CoAs or other yet unidentified (L-3-hydroxy-) acyl-CoAs derived molecules could represent metabolic signals that stimulate insulin secretion in a $\mathrm{K}^{+}$ATP independent way.

Considering that the nonsense mutation R236X leads to a protein lacking an important portion of the C-terminal domain, involved in subunit dimerization (10), it is reasonable to assume that a non-functional protein is generated. An easy tool to verify this hypothesis would have been to measure HADH enzyme activity in skin fibroblasts. However, the proband's parents refused to give their consent for skin biopsy.

In summary, we report the case of a new $H A D H$ gene R236X mutation in a hyperinsulinemic Italian patient with a diffuse form of HI. High-density microarray analysis revealed $H A D H$ gene to be a likely candidate in this patient and direct sequencing confirmed the presence of causative mutation.

This case indicates that mutations of $H A D H$ gene should be sought in hyperinsulinemic patients in whom a diffuse form of $\mathrm{HI}$ and autosomal recessive inheritance can be presumed when KCNJ11 and ABCC8 genes mutational screening is negative, independently from the organic acids and acylcarnitine metabolism.

\section{Declaration of interest}

The authors do not have any conflict of interest that could be perceived as prejudicing the impartiality of the research reported.

\section{Funding}

This work was supported by MIUR grants (FIRB 2004 internazionale and PRIN20063299) and University fund FIRST 2006. This study was carried out in the context of the $\mathrm{PhD}$ program of Molecular Medicine (University of Milan).

\section{Acknowledgements}

The authors acknowledge the precious help of Drs Laura Bosio, Maddalena Bove, Ilaria Zamproni, Roberta Spinelli, and Cristina Battaglia. We thank LABOGEN S.r.l. for sequencing technical support.

\section{References}

1 Hussain K. Diagnosis and management of hyperinsulinaemic hypoglycaemia of infancy. Hormone Research 200869 2-13.

2 Molven A, Matre GE, Duran M, Wanders RJ, Rishaug U, Njølstad PR, Jellum E \& Søvik O. Familial hyperinsulinemic hypoglycemia caused by a defect in the SCHAD enzyme of mitochondrial fatty acid oxidation. Diabetes 200453 221-227.

3 Clayton PT, Eaton S, Aynsley-Green A, Edginton M, Hussain K, Krywawych S, Datta V, Malingre HE, Berger R \& van den Berg IE. Hyperinsulinism in short-chain L-3-hydroxyacyl-CoA dehydrogenase deficiency reveals the importance of betaoxidation in insulin secretion. Journal of Clinical Investigation 2001108 457-465.

4 Hussain K, Clayton PT, Krywawych S, Chatziandreou I, Mills P, Ginbey DW, Geboers AJ, Berger R, van den Berg IE \& Eaton S. 
Hyperinsulinism of infancy associated with a novel splice site mutation in the SCHAD gene. Journal of Pediatrics 2005 146 706-708.

5 Biagiotti L, Proverbio MC, Bosio L, Gervasi F, Rovida E, Cerioni V, Bove M, Valin PS, Albarello L, Zamproni I, Grassi S, Doglioni C, Mora S, Chiumello G \& Biunno I. Identification of two novel frameshift mutations in the KCNJ11 gene in two Italian patients affected by congenital hyperinsulinism of infancy. Experimental and Molecular Pathology 200783 59-64.

6 Otonkoski T, Näntö-Salonen K, Seppanen M, Veijola R, Huopio H, Hussain K, Tapanainen P, Eskola O, Parkkola R, Extröm K, Guiot Y, Rahier J, Laakso M, Rintala R, Muutila P \& Minn H. Noninvasive diagnosis of focal hyperinsulinism of infancy with $\left[{ }^{18} \mathrm{~F}\right]-\mathrm{DOPA}$ positron emission tomography. Diabetes 200655 13-18.

7 Martens GA, Vervoort A, Van de Casteele M, Stangé G, Hellemans K, Van Thi HV, Schuit F \& Pipeleers D. Specificity in beta cell expression of L-3-hydroxyacyl-CoA dehydrogenase, short chain, and potential role in down-regulating insulin release. Journal of Biological Chemistry 2007282 21134-21144.
8 James C, Kapoor R, Eaton S, Flanaghan S, Ellard S \& Hussain K. Protein sensitive hyperinsulinemic hypoglycemia due to a novel mutation in the short-chain L-3-hydroxyacyl-CoA dehydrogenase (HADH) gene with normal acylcarnitines and urine organic acids. Hormone Research 200870 S53.

9 Prentki M, Vischer S, Glennon MC, Regazzi R, Deeney JT \& Corkey BE. Malonyl-CoA and long chain acyl-CoA esters as metabolic coupling factors in nutrient-induced insulin secretion. Journal of Biological Chemistry 1992267 5802-5810.

10 Barycki JJ, O'Brien LK, Bratt JM, Zhang R, Sanishvili R, Strauss AW \& Banaszak LJ. Biochemical characterization and crystal structure determination of human heart short chain L-3hydroxyacyl-CoA dehydrogenase provide insights into catalytic mechanism. Biochemistry 199938 5786-5798.

Received 18 March 2009

Accepted 23 March 2009 